$\underline{\text { RESEÑA }}$

\title{
I Seminario de investigación histórica "Dagli archivi alla ricerca. Confronto per una nuova stagione di studi sulla massoneria" (Centro Ricerche Storiche sulla Libera Muratoria, Turín, 2017)
}

Fernando M. Anaya-Gámez

Universidad de Málaga, España fanagam@gmail.com

Recepción: 20 de abril de 2018/ Aceptación: 23 de mayo de 2018 doi: https://doi.org/10.15517/rehmlac.v10i1.33414

"El único deber que tenemos con la historia es el de escribirla de nuevo" Oscar Wilde

\section{Introducción}

Decía el excelso hombre de las letras italianas Cesare Pavese, un pensamiento que encierra en sí la esencia de la propia vida: "Busca en el hombre pobre las virtudes del rico (exquisitez, sentimientos delicados, sociabilidad...) y en el rico las virtudes del pobre (seriedad, pragmatismo sencillo, bondad laboriosa...)". Y si su íntegra visión la extrapolamos al mundo científico, podríamos imaginar un resultado honesto y de múltiples beneficios para todos. En sí, el profesor Marco Novarino ${ }^{1}$ lo logró ampliamente no solo en los esfuerzos por conseguir un foro intelectual y académico en el sentido más estricto de su vínculo con la sana discusión de ideas, e inclusive en la remembranza de la categoría histórica que contiene el propio vocablo, sino porque además procuró estrechar

\footnotetext{
${ }^{1}$ El profesor Marco Novarino está vinculado a la Universidad de Turín y es director del Centro di Ricerche Storiche sulla Libera-Muratoria de dicha ciudad. Su excelente trayectoria de trabajo no solo le valió cosechar una amplia producción científica (inclusive galardonada) sino que además se ha ganado de pleno derecho el reconocimiento de la comunidad de historiadores que versan su trabajo sobre la masonería, especialmente en su exploración por las relaciones entre Italia y España. Además de ser miembro del Centro de Estudios Históricos de la Masonería Española (CEHME), pertenece a otras sociedades y entidades, respaldadas por su membresía en publicaciones científicas de notable reconocimiento. Véase parte de su senda científica en: "Marco Novarino", Spagna Contemporanea, http://www.spagnacontemporanea.it $/$ index.php?option $=$ com content\&view $=$ article\&id $=152 \& I t e m i d=86 \& l a n$ $\mathrm{g}=\mathrm{it}$
} 
ampliamente los lazos entre todo tipo de paradigmas multidisciplinares ante el objetivo común de los congregados: una gran devoción por la historiografía en torno a la masonería. Y es que la gran acogida del Centro Ricerche Storiche sulla Libera Muratoria ${ }^{2}$ en su primer seminario de investigación histórica basado en la fórmula de congregar a grandes referentes de la historiografía dedicada a los estudios sobre las masonerías junto a noveles investigadores, responde a la perfección a la sexta acepción que se indica en el diccionario de la Real Academia Española de la Lengua. Pero además, cumple con una de las funciones primordiales del propio centro de investigación: la organización de eventos científicos sobre los temas acordados de forma estatutaria.

Por todo ello, esta primera edición titulada Dagli archivi alla ricerca. Confronto per una nuova stagione di studi sulla massoneria, y que se desarrolló en el propio centro durante el día 27 de octubre de 2017, se proyectó con eficiente sabiduría y abundantes resultados, dando lugar a la narración que compila brevemente lo que aconteció y se presenta en esta reseña.

\section{Un foro, panoplia de ideas}

Ciertamente se cumplen veinte años de la fundación del Centro di Ricerche Storiche sulla Libera-Muratoria (CRSL-M), desde su creación en 1997 por los profesores Augusto Comba y Marco Novarino. Y fiel a su regreso a la activad desde el 2011, gracias al apoyo del Collegio Circoscrizionale Piemonte e Valle d'Aosta y del Consiglio torinese dei Maestri Venerabili del Grande Oriente d'Italia, ha ido desarrollando una intensa actividad vinculada a sus principios fundacionales: gestionar y ampliar su archivo mediante la adquisición, digitalización y catalogación de sus fondos, extensible a su biblioteca especializada en masonería y esoterismo, y posibilitando así su consulta, junto a la idea de posibilitar el estudio y la investigación mediante acuerdos con entidades universitarias y la realización de eventos científicos. Motivación esta última que, como hemos comentado con anterioridad, ha propiciado este encuentro internacional coordinado por su director científico, el profesor Marco Novarino, junto a Daniele Lanzavecchia (presidente del Consiglio torinese dei Maestri Venerabili), Renato Lavarini (presidente del Collegio Circoscrizionale Piemonte e Valle d'Aosta), y los colaboradores del centro: la profesora Emanuela Locci junto a los investigadores Demetrio Xoccato y Enrico Miletto. Sobre este particular, la sesión de trabajo se dividió en dos partes fundamentales: la exposición de la labor científica desarrollada por parte de los jóvenes investigadores junto a una conferencia plenaria, y su posterior debate en unión al comité científico. Dicha comisión estuvo conformada por los profesores: Pierre-Yves Beaurepaire (Universidad de Niza), Gian Mario Cazzaniga

\footnotetext{
${ }^{2}$ Véase, para ampliar más información sobre sus actividades, "Centro di Ricerche Storiche sulla Libera-
} Muratoria", Centro di Ricerche Storiche sulla Libera-Muratoria, http://www.crsl-m.org/ 
(Universidad de Pisa), Fulvio Conti (Universidad de Florencia), Marco Cuzzi (Universidad de Milán), Ricardo Martínez Esquivel (Universidad de Costa Rica), Santi Fedele (Universidad de Mesina), José Antonio Ferrer Benimeli (Universidad de Zaragoza), Luis P. Martín (Universidad de Pau et des Pays de l'Adour) y Rosalino Sacchi (Universidad de Turín). Es por todo ello que la siguiente narración intenta dibujar grosso modo, y en el mismo orden de actuación, las distintas aportaciones de sus miembros.

Comenzó la actividad, en su primera parte, el doctorando Fernando Miguel Anaya Gámez ${ }^{3}$, adscrito a la Universidad de Málaga y miembro del Centro de Estudios Históricos de la Masonería Española (CEHME), el cual basó su alocución en las consignas establecidas por la organización para todos los participantes: breve exposición del trabajo desarrollado, producción más significativa hasta el momento y los proyectos futuros que se piensan acometer. En este sentido, el ponente nos participó de su experiencia inicial ${ }^{4}$ dentro del Máster en Historia de la Masonería en España ${ }^{5}$ de la Universidad Nacional de Educación a Distancia (UNED), lo cual le permitió aproximarse con mayor profundidad al tema vertebral que circundan sus investigaciones actuales: las relaciones entre música, masonería y sociabilidad. Tras exponer brevemente la casuística histórica en vínculo a las masonerías que históricamente se congregaron en el territorio español, hizo lo propio con la evolución de los estudios que sobre música y masonería, con especial atención a lo desarrollado desde Italia $^{6}$, para adentrarse en sus trabajos ${ }^{7}$ y próximas producciones científicas que van viendo la luz, y finalmente significar tres amplios deseos de futuro: junto con la finalización de su tesis doctoral, sigue persiguiendo la intención de crear un grupo internacional sobre arte y masonería, el cual podría instaurar una base de datos sobre artistas masones y su producción.

\footnotetext{
${ }^{3}$ Su perfil científico se puede consultar a través de dos enlaces ampliamente utilizados en la actualidad (de los múltiples existentes): véanse, "Fernando M. Anaya-Gámez", Academia.edu, https://uma.academia.edu/FernandoAnayaG\%C3\%A1mez, y "Fernando M. Anaya-Gámez", Orcid, https://orcid.org/0000-0002-3147-7229

${ }^{4}$ Fernando M. Anaya-Gámez, "Música y masonería: prontuario para un estado de la cuestión" REHMLAC+ 6, no. 1 (mayo-noviembre 2014): 141-145, doi: http://dx.doi.org/10.15517/rehmlac.v6i1.15231

5 Véase: "Máster en historia de la masonería", UNED, http://www2.uned.es/dptohdi/master/modular_mov_asociativos.htm

${ }^{6}$ Dos claros ejemplos serían los monográficos realizados en su día por los profesores Basso y Tocchini. Véanse: Alberto Basso, L'invenzione della gioia. Musica e massoneria nell'età dei Lumi (Milán: Garzanti, 1994) y Gerardo Tocchini, I Fratelli d'Orfeo. Gluck e il teatro musicale massonico tra Vienna e Parigi, (Florencia: Leo S. Olschki, 1998).

${ }^{7}$ Véase, Anaya-Gámez, "Y la lira volvió a sonar: breve estudio sobre las relaciones semánticas entre música y masonería", en La masonería hispano-lusa: de los absolutismos a las democracias. XIV Symposium Internacional de Historia de la Masonería Española, coords. José Miguel Delgado Idarreta e Yván Pozuelo Andrés, (Oviedo: Universidad de Oviedo y CEHME, 2017). Estudio también publicado en Anaya-Gámez, "Y la lira volvió a sonar: breve estudio sobre las relaciones semánticas entre música y masonería", en 300 años: Masonerias y masones (1717-2017). Tomo III: Artes, eds. Ricardo Martínez Esquivel, Yván Pozuelo y Rogelio Aragón (Ciudad de México: Palabra de Clío, 2017). Desde abril de 2018, y por el momento, se encuentra disponible en: http://www.palabradeclio.com.mx/pdf/detalle/300_tomo3
} 
En segundo lugar, fue el investigador Joachim Berger ${ }^{8}$ el que procedió a realizar su presentación en relación a la revista científica en la que participa. Y es que este doctor, además de ser coordinador de investigación del Leibniz Institute of European History ${ }^{9}$ de Mainz, y académico correspondiente de la Wissenschaftliche Gesellschaft für Theologie, forma parte del equipo editorial de la revista Journal for Research into Freemasonry and Fraternalism $^{10}$ desde el 2016, publicación especializada en las investigaciones históricas sobre la masonería y las sociedades fraternales. Conectada con el ámbito anglosajón, tiene entre sus principios el de promover la interdisciplinariedad académica en favor de la cultura material y la historia, desde la edad media hasta la actualidad, y en convivencia con el interés por las instituciones masónicas y su ámbito de aplicación local, regional, nacional e internacional. Por otro lado, y a nivel individual, Berger mostró su interés en el concepto de humanidad $^{11}$, haciendo mención a su actual proyecto de trabajo ${ }^{12}$ que sigue esa línea argumental.

Por otro lado, la doctora Nicoletta Casano, colaboradora científica del Centre Interdisciplinaire d'Étude des Religions et de la Laïcité (CIERL), centro de investigación de la Universidad Libre de Bruselas, nos habló de sus estudios en vinculación al área principal a la que circunscribió su tesis doctoral ${ }^{13}$, en conjunción con otras vertientes o sendas de indagaciones relevantes. Ciertamente, su trabajo se articula, como ella misma nos aclaró, en torno a las líneas abiertas por los profesores Santi Fedele ${ }^{14}$ (Universidad de Mesina) y Anne Morelli ${ }^{15}$ (Universidad Libre de Bruselas), en consonancia con lo que se

\footnotetext{
${ }^{8}$ Véase su perfil científico para recabar más información en: “Joachim Berger”, Academia.edu, http://iegmainz.academia.edu/JoachimBerger

${ }^{9}$ Para mayor información: véase, “Joachim Berger", IEG, http://www.ieg-mainz.de/en/institute/people/berger 10 Véase, Journal for Research into Freemasonry and Fraternalism, Equinox, https://journals.equinoxpub.com/index.php/JRFF

${ }^{11}$ Véase, Joachim Berger, “"Une œuvre internationale d'un caractère humanitaire»: The Appeal to Humanity in International Masonic Relations", en Humanity. A History of European Concepts in Practice From the Sixteenth Century to the Present, eds. Fabian Klose, Mirjam Thulin (Göttingen: Vandenhoeck \& Ruprecht, 2016), 231-248.

12 Para mayor concreción del proyecto comentado, véase, "For God, Fatherland, and Humanity - Masonic Internationalism in Europe, c. 1850-1935”, IEG, http://www.ieg-mainz.de/en/researchprojects/masonic internationalism_in_europe 1850-1935

${ }_{13}$ Véase, Nicoletta Casano, "Les réseaux unissant francs-maçons et laïques belges et italiens de la fin du XIXe siècle jusqu'à la Deuxième guerre mondiale. Prémisses et réalisation de l'accueil en Belgique des fuorusciti italiens", Revue Belge d'Historie Contemporaine XLIII, no. 2/3 (2013): 238-240, https://www.journalbelgianhistory.be/fr/journal/belgisch-tijdschrift-voor-nieuwste-geschiedenis-xliii-2013-23/r-seaux-unissant-francs-ma

${ }^{14}$ Es interesante reseñar al respecto tres monografías destacadas, entre su amplio currículo investigador. Véanse, Santi Fedele, La Massoneria italiana nell'esilio e nella clandestinità 1927-1939 (Milán: Franco Angeli, 2005); Alessandro Tedeschi Gran Maestro dell'esilio (Bolonia: Percosi, 2008); y La Massoneria italiana tra Otto e Novecento (Foggia: Bastogi Editrice Italiana, 2011).

${ }^{15}$ Entre sus distintos trabajos científicos acometidos, y en mención expresa al discurso que se viene hilando, véanse por ejemplo: Anne Morelli, La participation des émigrées italiens à la résistance belge (Roma:
} 
podría denominar como la diáspora de masones y librepensadores italianos que, durante el régimen de Mussolini, optaron por refugiarse en Francia y en Bélgica para acceder a la pura expresión de sus ideales de pacifismo y en un claro bloque antifascista ${ }^{16}$. Particularmente, su obra Libres et persécutés. Francs-maçons et lä̈ques italiens en exil pedant le fascisme ${ }^{17}$ vino a compendiar los resultados obtenidos en suma con sus artículos científicos publicados ${ }^{18}$. Igualmente, nos abre la puerta a otra directriz investigadora en donde la mujer masona belga es la protagonista en su evolución histórica, concretándose en el análisis de varias agrupaciones (logias y obediencias) determinadas; de hecho, y a día de hoy, podemos contemplar su colaboración en la exposición temporal Les femmes frappent à la porte du temple, 1717-2017 ${ }^{19}$ del Museo Belga de la Masonería, institución con la que mantiene vínculo. Indudablemente, una importante senda de trabajo que complementa lo que se viene realizando en otros países ${ }^{20}$ al respecto.

El doctorando Felipe Côrte Real de Camargo, vinculado a la Universidad de Bristol y miembro del Correspondence Circle of the Quatuor Coronati Lodge no 2076, nos habló de sus intereses investigadores que entroncan directamente con un emergente punto de vista de gran interés: el patrimonio material masónico en unión a la denominada tradición esotérica occidental. Focalizando su atención principal en la riqueza simbólica de los mandiles, su línea argumental se adentra, en propias palabras del investigador, en la "recepción y resignificación" de los elementos hermético-cabalísticos y alquímicos -será interesante aproximarse a los resultados de sus futuras investigaciones sobre este paradigma en la estancia prevista en la Biblioteca Filosófica Hermética o The Ritman Library de

Ministerio Affari Esteri, 1983) y Fascismo e antifascismo nell'emigrazione italiana in Belgio: 1922-1940 (Turín: Bonacci, 1987)

${ }^{16}$ Sobre este aspecto concreto podemos exhibir los trabajos desarrollados por el profesor Luis P. Martín y, con mayor amplitud, el profesor Aldo Mola. Véanse, como elemento ejemplificador y en breve detalle, Luis P. Martín Martínez, "La Asociación Masónica Internacional (1901-1940) o la utopía pacifista", en $L a$ masonería en la España del siglo XX. VII Symposium Internacional de Historia de la Masonería Española, coord. José Antonio Ferrer Benimeli (Zaragoza: CEHME, Universidad de Castilla-La Mancha, Cortes de Castilla-La Mancha, 1996), Tomo I, 1115-1128; y Aldo Alessandro Mola, "Las relaciones masónicas italianoespañolas y la Avocación Masónica Internacional", en La masonería en la España del siglo XX. VII Symposium Internacional de Historia de la Masonería Española, coord. Ferrer Benimeli (Zaragoza: CEHME, Universidad de Castilla-La Mancha, Cortes de Castilla-La Mancha, 1996), Tomo I, 471-488.

17 Casano, Libres et persécutés. Francs-maçons et laïques italiens en exil pedant le fascisme (París: Classiques Garnier, 2016).

18 Véase su perfil científico para recabar más información en: "Nicoletta Casano", Academia.edu, http://ulb.academia.edu/NicolettaCasano

19 Véase, en este sentido, "Les femmes frappent à la porte du temple, 1717-2017", Museo Belga de la Masonería, http://mbfm.be/wp/fr/expositions-temporaires/

${ }^{20}$ Es interesante resaltar, como ya nos hemos hecho eco en anteriores ocasiones, de los trabajos de Snoek, Révauger, Locci, Ortiz, Lacalzada, Hottinger, Elovskikh, Alba, entre otras y otros, junto con el destacado monográfico aportado en la reciente publicación que se apunta a continuación: véase, VV.AA., en 300 años: Masonerías y masones (1717-2017). Tomo IV: Exclusión, eds. Martínez Esquivel, Pozuelo y Aragón (Ciudad de México: Palabra de Clío, 2018). Desde abril de 2018, y por el momento, se encuentra disponible en: http://www.palabradeclio.com.mx/pdf/detalle/300 tomo4 
Ámsterdam (Holanda)-, evidenciando lo destacable de este tipo de fuentes significantes ${ }^{21}$ en conexión con los momentos fundacionales de la masonería (siglo XVIII), para desarrollar un punto de partida extensible al patrimonio cultural e histórico de la propia institución. Y aunque no podemos obviar ni su extenso conocimiento sobre urbanismo, arquitectura de la ciudad y su historia cultural, e inclusive en la vertiente de las relaciones internacionales de la masonería ${ }^{22}$, estaremos expectantes ante la continuación ${ }^{23}$ de sus presentes pesquisas ${ }^{24}$.

Vinculada al Collegio Ghislieri y a la Universidad Ca'Foscari de Venecia, la doctora Giulia Delogu disertó sobre la unión entre masonería y literatura ${ }^{25}$ en el siglo XVIII y los inicios del XIX. Una senda investigadora que ya exploró tanto en su tesis doctoral ${ }^{26}$ como en trabajos anteriores ${ }^{27}$ y que queda refrendada por sendas monografías publicadas ${ }^{28}$ : La poetica della virtù. Comunicazione política e rappresentazione del potere in Italia tra

${ }^{21}$ Véase sobre esta senda discursiva, Felipe Côrte Real de Camargo, "Una mirada distinta a las fuentes en la historia de la masonería", REHMLAC+ 8, no. 2 (diciembre 2016-abril 2017): 307-310, doi: http://dx.doi.org/10.15517/rehmlac.v8i2.27252

${ }^{22}$ Véanse, Côrte Real de Camargo, "Protect the Integrity: regularidade no discurso das relações maçônicas internacionais entre Brasil e Inglaterra (1880 - 2000)", REHMLAC+ 8, no. 1 (mayo 2016-noviembre 2016): 130-150, doi: https://doi.org/10.15517/rehmlac.v8i1.24276; y la ponencia del mismo autor, que de momento no está publicada, "Dependence and Autonomy: The English Freemasons and their Relations in Argentina and Brazil (1859-1935)" (presentada en World Conference on Fraternalism, Freemasonry \& History: Research in Ritual, Secrecy and Civil Society, París, 26 y 27 de mayo de 2017), https://revistas.ucr.ac.cr/index.php/rehmlac/article/view/31387/31670

${ }^{23}$ Véase, Côrte Real de Camargo, "La cultura material como fuente para la historia de la masonería: el caso de los mandiles de la masonería inglesa preunificación (1720's-1820's)", en 300 años: Masonerías y masones (1717-2017). Tomo V: Cosmopolitismos, eds. Martínez Esquivel, Pozuelo y Aragón (Ciudad de México: Palabra de Clío, 2018). Desde abril de 2018, y por el momento, se encuentra disponible en: http://www.palabradeclio.com.mx/pdf/detalle/300 tomo5

${ }_{24}$ Véase su perfil científico para recabar más información en: "Felipe Côrte Real de Camargo", Academia.edu, http://bristol.academia.edu/FelipeC $\%$ C3\%B4rteReal

${ }^{25}$ Recuérdese que la unión entre literatura y masonería se viene articulando desde hace años y en conexión con una variada paleta de focos de acción e intención. Son reseñables los trabajos de Parada, Corbalán, Mercier, Batley, Prescott, Leighton, Mulvey-Roberts, Önnerfors, Gilbert, entre otros. Igualmente, destaca el monográfico que presentó esta revista en atención a las relaciones entre masonería, literatura y escritor; véase: https://revistas.ucr.ac.cr/index.php/rehmlac/issue/view/1346. Y finalmente, no se puede obviar los trabajos realizados por el historiador y escritor Ricardo Serna, además de la reciente lectura de su tesis doctoral: Ricardo Serna Galindo, "Literatura y ámbito masónico. A propósito de la novela Pequeñeces, del jesuita Luis Coloma" (Tesis de doctorado en patrimonio, Universidad de Jaén, 2017). Véase, sobre esta última referencia: https://revistas.ucr.ac.cr/index.php/rehmlac/article/view/30053/31665

${ }^{26}$ Giulia Delogu, "Trieste «di tesori e virtù sede gioconda». Una storia poetica, dall'Arcadia RomanoSonziaca alla Società di Minerva" (Tesis de doctorado en ciencias humanísticas, Universidad de Trieste, 2015). Véase su trabajo doctoral en el siguiente enlace: https://www.openstarts.units.it/bitstream/10077/11002/1/TesiDelogu.pdf

27 Véase su perfil científico para recabar más información en: "Giulia Delogu", Academia.edu, https://unive.academia.edu/GiuliaDelogu

${ }^{28}$ Giulia Delogu, La poetica della virtù. Comunicazione política e rappresentazione del potere in Italia tra Sette e Ottocento (Milán - Udine: Mimesis, 2017) y «Di virtù lira sonante». Poesia e massoneria in Italia tra Settecento e Primo Ottocento (Pavía: Varini Publishing, 2014). 
Sette e Ottocento y «Di virtù lira sonante». Poesia e massoneria in Italia tra Settecento e Primo Ottocento. Y es que la palabra oral o escrita va más allá de los medios o soportes en la que se plasman y viaja hasta la mente de los que la reciben, siendo por tanto de especial realce la difusión del mensaje masónico a través de su línea de investigación centrada en la Italia de los siglos XVIII y principios del XIX, junto con el estudio del concepto "virtud" en combinación con las ideas políticas de perfección, educación e ideales masónicos, a través de los pensamientos de Longano, Grimaldi, Filangieri y Pagano. Igualmente, y siguiendo el trazado de la comunicación política, será interesante aproximarnos en un próximo futuro a su participación en el proyecto: Networks of Power and Communication: Mapping Napoleon in Europe.

Además de interesarse por la historia local de su Cosenza natal y por cuestiones de antropología cultural y religiosa, el profesor y doctorando Luca Fragale ${ }^{29}$, adscrito al Departamento de Ciencias Políticas de la Universidad de Roma-La Sapienza, nos participó de su interés por el tema que explora sus estudios doctorales: la acción de los miembros del Parlamento de Italia vinculados a la masonería durante el período que transcurrió entre la denominada Marcha sobre Roma y la controvertida publicación de la Ley de asociaciones (como parte de las Leyes fascistísimas) de 1925. Sin duda, una senda de análisis explorada con amplitud por el enorme trabajo prolífico del profesor Fulvio Conti ${ }^{30}$, supervisor de los trabajos doctorales del ponente, y que abre la posibilidad de seguir rastreando esa etapa histórica. De la misma forma, y sin olvidar su aproximación a la figura del filósofo y matemático Arturo Reghini, Fragale nos brindó la oportunidad de acercarnos a sus próximas pesquisas sobre la intervención de la masonería en el rescate de los judíos en un ámbito concreto, dada su adhesión al proyecto: Freemasonry, Resistance and the Rescue of the Jews in Denmark - 1943: an overview about involvements and missing links ${ }^{31}$.

Impresiona atisbar el trabajo que ha desarrollado el ingeniero informático y doctorando en historia moderna Thomas Fressin ${ }^{32}$ en torno a su incalculable aportación al panorama emergente de las denominadas "humanidades digitales". Hablamos, y en eso estableció parte de su presentación, de dos recursos importantes en los que trabajó tras su paso por la Biblioteca Nacional de Francia, en su sección de Manuscritos: por un lado,

29 Véase su perfil científico para recabar más información en: "Luca Irwin Fragale", Academia.edu, https://wwwuniroma1.academia.edu/LucaIrwinFragale

${ }^{30}$ Seleccionar obras de referencia entre la ingente labor historiográfica del profesor Conti sería materia de un artículo de por sí. Por ello, incluyendo su participación en dos ediciones de los encuentros internacionales del CEHME y sin menospreciar el resto, hemos seleccionado algunos monográficos más actuales junto con una obra que atañe directamente al foco de estudio comentado. Véanse, Fulvio Conti, Italia immaginata. Sentimenti, memorie e politica fra Otto e Novecento (Pisa: Pacini, 2017); Massoneria e religioni civil. Cultura laica e liturgie politiche fra XVIII e XX secolo (Bolonia: Il Mulino, 2008); y Storia della massoneria italiana. Dal Risorgimento al fascismo (Bolonia: Il Mulino, 2003).

${ }^{31}$ Más información en su perfil académico antes reseñado.

${ }^{32}$ Véase su perfil científico para recabar más información en su página personal: "Thomas Fressin”, Thomas Fressin, http://tfressin.fr/ 
Gallica Alerts $^{33}$, como herramienta que manda alertas sobre las digitalizaciones que progresivamente se van realizando desde Gallica (la versión digital de la referida biblioteca); y el Bossu Record ${ }^{34}$, como forma de acceso a los registros biográficos de masones - hablamos de cerca de ciento sesenta y seis mil fichas- que se recogen de 1780 a 1850 en dicha institución, a partir del fichero ${ }^{35}$ creado por Jean Bossu $^{36}$ (1911-1985). Y es que además de ser el presidente de la recientemente constituida Société Française d'Histoire des Sociétés Fraternelles (SFHSF) ${ }^{37}$, ser el director de la publicación L'Archer Français $^{38}$, y de formar parte de varias logias de investigación en vínculo con la indagación en las sociedades paramasónicas, Fressin también se incorpora a la senda investigadora a través de sus trabajos doctorales, proyectando su interés hacia los denominados Nobles jeux ${ }^{39}$, en la práctica del arco, la ballesta y el arcabuz por la milicia urbana en los contextos de sociabilidad y en aproximación a la masonería (observando inclusive si hay alguna práctica ritualista), siguiendo una de las líneas abiertas por su director de tesis, el profesor Pierre-Yves Beaurepaire ${ }^{40}$. No es de extrañar por tanto que, además de su creciente interés por la sociabilidad en la historia ${ }^{41}$, el investigador Thomas Fressin visualice un deseo de futuro que abarque un proyecto internacional sobre la creación de un archivo en línea que contenga manuscritos y libros digitalizados sobre la historia de la masonería, con acceso a los investigadores (en sus distintas vertientes), y previo acuerdo con archivos, bibliotecas, centros de investigación y universidades.

Exitosas son las dos trayectorias científicas instauradas por la doctora Emanuela Locci $^{42}$ en vínculo con su Mediterráneo natal. Dualidad sobre la cual nos disertó y que le llevó a explorar cómo la masonería se extendió por Egipto, Túnez, Malta y Turquía ${ }^{43}$, en

\footnotetext{
${ }^{33}$ Véase, "Gallica Alerts", Gallica Alerts, http://gallica-alertes.fr/

${ }^{34}$ Véase, "Bossu Record", Le fichier Bossu, http://fichier-bossu.fr/

${ }^{35}$ Véase, "Le fichier Bossu", Le fichier Bossu, http://fichier-bossu.fr/more.php

36 Véase, para más información sobre su figura, "Jean Bossu (1911-1985)", La Maçonne, http://lamaconne.over-blog.com/2016/05/jean-bossu-1911-1985.html

${ }^{37}$ Para más información, véase, "Société Française d'Historie des Sociétés Fraternelles", Société Française d'Historie des Sociétés Fraternelles, https://sfhsf.hypotheses.org/

${ }^{38}$ Véase, "L’Archer Français", L'Archer Français, http://larcherfrancais.fr/

${ }^{39}$ Lo que podría traducirse como los "juegos de nobleza" (o también conocidos como los juegos de papegai o papegant) es una forma de entretenimiento de las clases sociales acomodadas. Uno de los investigadores más relevantes que ha investigado sobre esta práctica de sociabilidad ha sido Pierre-Yves Beaurepaire.

40 Entre la excepcional producción científica del profesor Beaurepaire, destaca la siguiente referencia entroncada directamente con el argumento que venimos exponiendo. Véase, Pierre-Yves Beaurepaire, Nobles jeux de l'Arc et loges maçonniques dans la France des Lumières (Montmorency: Ivore-clair, 2002).

${ }^{41}$ Véase su reciente monografía, Grand Armorial des Chevaliers Bienfaisants de la Cité Sainte (París: autoedición, 2017). Más información en: "Grand Armorial des Chevaliers Bienfaisants de la Cité Sainte", Grand Armorial des Chevaliers Bienfaisants de la Cité Sainte, http://www.grand-armorial-cbcs.fr/

42 Véase su perfil científico para recabar más información en: "Emanuela Locci", Academia.edu, https://independent.academia.edu/ManuelaLocci

43 Véase, a tal afecto, Emanuela Locci, Massoneria nel Mediterraneo. Egitto, Tunisia e Malta (Roma: BastogiLibri, 2014).
} 
suma a efectuar un análisis sobre cómo ha sido la evolución histórica de la masonería femenina $^{44}$ en un estadio comparativo entre Italia, España, Francia, Inglaterra e igualmente Turquía. Adscrita como profesora de la Università degli Studi di Torino, siendo además investigadora del Centro Studi Mediterraneo al-Mutawassit y colaboradora del Centro Ricerche Storiche sulla Libera Muratoria, sus inquietudes por el estudio histórico de la masonería las plasmó a través de su tesis al estrechar lazos con el Imperio otomano ${ }^{45}$, siguiendo además ejemplo vivo de lo que mencionó en el resumen de su artículo ${ }^{46}$ para esta revista en su anterior número: "De gran importancia es la propensión italiana a mezclarse con la población local, para que la comunidad italiana no sea percibida como un cuerpo extraño, sino como un complemento a la sociedad de acogida", perspectiva vital que le valió adentrarse en la otra orilla mediterránea ${ }^{47}$ que aún queda por analizar en profundidad. Igualmente, son ampliamente destacables sus aportaciones recientes ${ }^{48}$ y las futuras ${ }^{49}$ en el propósito de la difusión de la historiografía del Gran Oriente de Italia.

Junto al doctor Luca G. Manenti nos aproximamos a su concepción investigadora ${ }^{50}$ en consonancia con el estudio historiográfico de la masonería en Trieste y, fundamentalmente, con la extensa red de sociabilidad que discurrió entre los hermanos francmasones unidos a la idea del irredentismo ${ }^{51}$ en Italia. De hecho, el profesor Manenti, vinculado tanto a la Università degli Studi di Trieste, y siendo además miembro del consejo directivo del Istituto Regionale per la Storia del Movimiento di Liberazione nel Friuli Venezia Giulia (Irsml FVG), resaltó con sus palabras la importancia que tuvo la masonería

\footnotetext{
${ }^{44}$ Además de las breves alusiones comentadas al comienzo de esta reseña, véase, Locci, Storia della massoneria femminile. Dalle corporazioni alle obbedienze (Roma: BastogiLibri, 2017).

${ }^{45}$ Planteamiento investigador que quedó plasmado en el siguiente libro: véase, Locci, Il cammino di Hiram. La massoneria nell'Imperio Ottomano (Foggia: Bastogi Editrice Italiana, 2013).

46 Locci, "Las logias italianas en Turquía: entre el fortalecimiento de la comunidad italiana y el cosmopolitismo", REHMLAC+ 9, no. 2 (diciembre 2017-abril 2018): 47-55, https://revistas.ucr.ac.cr/index.php/rehmlac/article/view/30729/31656

${ }^{47}$ De gran proyección e interés son también los trabajos que la doctoranda Valeria Aguiar Bobet se encuentra desarrollando actualmente en alusión a su tesis doctoral y en vínculo con la masonería en Marruecos. Para mayor información, véase su perfil científico para recabar más información en: "Valeria Aguiar Bobet", Academia.edu, https://uji.academia.edu/ValeriaAguiarBobet

${ }^{48}$ Véase, Locci, "The First Lodge in the Ottoman Empire: Oriental Lodge, 1856-1909", en Reflections on 300 years of freemasonry, ed. John S. Wade (Londres: Lewis Masonic, 2017), 411-418. Y también la ponencia, que de momento no está publicada, "Freemasonry in Libia" (presentada en World Conference on Fraternalism, Freemasonry \& History: Research in Ritual, Secrecy and Civil Society, París, 26 y 27 de mayo de 2017), https://revistas.ucr.ac.cr/index.php/rehmlac/article/view/31387/31670

${ }^{49}$ La profesora Locci nos comentó, al término de su alocución, su trabajo en curso sobre una monografía en inglés que versará sobre la historia de la masonería en Italia.

50 Véase su perfil científico para recabar más información en: "Luca G. Manenti", Academia.edu, https://units.academia.edu/LucaGManenti

${ }^{51}$ Véase, Luca G. Manenti, Massoneria e irredentismo. Geografia dell'associazionismo patriottico in Italia tra Otto e Novecento (Trieste: Irsml FVG, 2015).
} 
en las distintas acciones de resistencia ${ }^{52}$ (inclusive participando de la propaganda de forma decidida) en los períodos convulsos y belicistas de comienzos del siglo XX. Igualmente, incidió en la importancia que tiene tanto el estudio de la masonería en la búsqueda de nexos de unión con otras doctrinas como la espiritista, al igual que en la propia concepción y creación de la Unión Europea.

Las conexiones que existieron entre el asociacionismo laico de Turín y la masonería entre mediados del siglo XIX y principios del XX es la principal pauta de acción investigadora en el doctorando Enrico Miletto ${ }^{53}$. Colaborador del Centro Ricerche Storiche sulla Libera Muratoria, además presentó su vínculo de estudio que versa sobre la existencia de la Università Popolare di Torino, en el rango de fechas comprendido entre 1900 y 1930 , como principal motor cultural de la ciudad, hasta el advenimiento de la etapa fascista. Una doble vertiente que se ha visto reflejada como autor en su monografía “...la coltura per il popolo". L'Università Popolare di Torino (1900-1930) ${ }^{54}$ y su coautoría ${ }^{55}$ en "senza distinzione política e religiosa”. Repertorio bibliográfico e archivístico sull'associazionismo laico a Torino e provincia (1848-1925) ${ }^{56}$ y "senza distinzione politica e religiosa”, Repertorio bibliográfico e archivístico sull'associazionismo laico in Piemonte $(1848-1925)^{57}$, y que próximamente tendrá como resultado un nuevo libro que analice la labor de este asociacionismo piamontés sobre la acción solidaria contra el pauperismo y los niños abandonados, en el período referenciado.

El doctor Yván Pozuelo Andrés ${ }^{58}$, vice-secretario del Centro de Estudios Históricos de la Masonería Española (CEHME) y editor de la Revista de Estudios Históricos de la

\footnotetext{
${ }^{52}$ Véase su próxima publicación, Luca G. Manenti, Fratelli in armi. L'interventismo massonico nelle pagine de «L'Idea Democratica».

${ }^{53}$ Véase su currículo científico en línea a través de la siguiente referencia: "Enrico Miletto", Istituto Istruzione Superiore State Erasmo da Rotterdam, http://www.erasmonichelino.gov.it/attachments/article/171/CV\%20MILETTO.pdf

${ }^{54}$ Véase, Enrico Miletto, “...la coltura per il popolo”. L’Università Popolare di Torino (1900-1930) (Turín: Università Popolare di Torino Editore, 2013).

${ }^{55}$ Más información sobre las obras que se reseñan a continuación y su proceso de trabajo, en la siguiente referencia que se indica: véase, "Progetto per la costituzione di un archivio «centralizzato» e un Centro di Ricerca sull'Associazionismo Laico in Piemonte", Ricerche, http://www.centrostudicalamandrei.it/ricerche.html

${ }_{56}$ Véase, Enrico Miletto y Marco Novarino, "senza distinzione política e religiosa". Repertorio bibliográfico $e$ archivístico sull'associazionismo laico a Torino e provincia (1848-1925) (Turín: Centro Studi Piero Calamandrei, 2011). Disponible en línea: véase, "Storia della massoneria e dell'associazionismo laico in Piemonte", Studi e ricerche, http://www.crsl-m.org/pdf/Repertorio\%20Associazionismo\%20laico\%201.pdf 57 Véase, Enrico Miletto, Marco Novarino y Demetrio Xocato, Ssenza distinzione política e religiosa". Repertorio bibliográfico e archivístico sull'associazionismo laico in Piemonte (1848-1925) (Turín: Centro Studi Piero Calamandrei, 2013). Disponible en línea: véase, "Storia della massoneria e dell'associazionismo laico in Piemonte", Studi e ricerche, http://www.crslm.org/pdf/Repertorio\%20Associazionismo\%20laico\%202.pdf

58 Véase su perfil científico para recabar más información en: "Yván Pozuelo", Academia.edu, http://independent.academia.edu/Yv\%C3\%A1nPozuelo
} 
Masonería Latinoamericana y Caribeña plus (REHMLAC+) elaboró su ponencia, bajo su virtuoso estilo comunicativo, perfilando sus inicios en los estudios históricos sobre la masonería, para focalizar su atención en el desarrollo de sus proyectos actuales y futuros. Si bien su próxima meta se centrará en establecer las relaciones entre francmasonería y movimiento obrero, este joven investigador de dilatado currículo científico presentó su amplio espectro de temáticas escogidas, como él mismo enunció atendiendo a "los nombres simbólicos, el tema de la 'discreción', los puertos, la delación entre masones durante la represión franquista, la antimasonería liberal, o incluso coordinar un monográfico sobre la relación entre masonería y II República, o proponer formas diferentes de publicar nuestros conocimientos como a través de una guía histórica de la masonería ${ }^{59}$ dentro del límite de una región o una ciudad, o [...] coordinar cinco tomos con títulos no específicos a la historia de la masonería como fueron Migraciones, Silencios, Arte, Exclusiones y Cosmopolitismo ${ }^{60}$ ", a lo que habría que añadir su interés por la literatura ${ }^{61}$ y la educación ${ }^{62}$, o su clara vocación de servir de puente entre distintos núcleos de investigación e investigadores, su incansable actividad en la difusión de la historia de la masonería ${ }^{63}$ y su fidelidad incondicional a los acontecimientos históricos de Gijón ${ }^{64}$, ciudad marinera donde reside.

En el caso de la investigadora Eleonora Salina ${ }^{65}$, vinculada a la Universidad de Turín, su propuesta versó sobre un estudio comparativo entre el Gran Oriente de Italia (GOI) y las grandes obediencias anglosajonas en relación a tres dimensiones de estudio sociológico: a nivel interno, sobre sus imbricaciones externas y desde una óptica política. En sí, y como ella nos indicó, se basó en los planteamientos sociológicos ${ }^{66}$ defendidos por

\footnotetext{
${ }^{59}$ Véase, Pozuelo Andrés, Guía histórica de la masonería en Asturias (Oviedo: Masónica, 2015).

${ }^{60}$ El conjunto de las obras reseñadas se encuentran en la página de la editorial: véase, "Colección: 300 años. Masonería y masones (1717-017)", Palabra de Clío, http://www.palabradeclio.com.mx/

61 Se perfila de interés su aproximación al escritor británico Rudyard Kipling: véase, Pozuelo Andrés, "Kipling y su sorprendente primera novela", REHMLAC+ 5, no. 2 (diciembre 2013-abril 2014): 143-160, https://revistas.ucr.ac.cr/index.php/rehmlac/article/view/12939/12235

${ }^{62}$ Véase, Martínez Esquivel y Pozuelo Andrés, "Educación escolar y masonería: krausismo y laicidad entre España y Costa Rica a finales del siglo XIX”, REHMLAC+ 9, No. 1 (mayo-noviembre 2017): 127-145, doi: http://dx.doi.org/10.15517/rehmlac.v9i1.28631

${ }^{63}$ El profesor Pozuelo es director de la interesante colección "Historiadores de la masonería". Véase, "Historiadores de la masonería", Masónica.es, https://www.masonica.es/materia/historiadores-de-lamasoneria/?parent_subject $=3646$

${ }^{64}$ Según nos informó el propio investigador, en la actualidad se encuentra trabajando en la historia de la logia Jovellanos no. 1 de Gijón y sus veintisiete años de historia en la primera parte del siglo XX.

65 Véase su perfil científico para recabar más información en: "Eleonora Salina", Academia.edu, https://unito.academia.edu/EleonoraSalina

${ }^{66}$ Desde el punto de vista histórico, el profesor Ferrer Benimeli y el profesor Ayala ya contemplaron esta perspectiva sociológica tanto a nivel general como particular (en el caso español). Véanse, Ferrer Benimeli, "El modelo sociológico de la masonería", en Masonería, revolución y reacción. IV Symposium Internacional de Historia de la Masonería Española, coord. Ferrer Benimeli (Zaragoza: CEHME, 1990), Tomo II, 919-928, y José Antonio Ayala, "Sociología de la masonería en la región de Murcia”, en La masonería en la España
} 
el profesor James Scott Kenney ${ }^{67}$ de la Memorial University of Newfoundland sobre dos narrativas representativas de la masonería desarrollada por el GOI: como sociedad iniciática y filantrópica o como organización unida a un mundo profano de intereses de toda tipología. En este sentido versó su argumentación a través de su proyecto de investigación Dentro la massoneria. Una ricerca sull'Obbedienza del Grande Oriente d'Italia, y de tres categorías de personas: masones, masones que lo fueron y estudiosos de la masonería; y así determinar parámetros anexos a la creciente afiliación a las filas masónicas italianas. En definitiva, en la visión de Salina, se pretende determinar cuál sería la auto-representación y la hetero-representación en sus distintos focos de percepción y connotación.

Finalizaron las intervenciones del primer bloque con el investigador Demetrio Xoccato $^{68}$. En vínculo con la Università di Torino y siendo además colaborador del Centro di Ricerche Storiche sulla Libera-Muratoria (CRSL-M), siendo una pieza vital de este al contribuir con la digitalización de las fuentes existentes dentro de la institución, su ponencia se centró, desde su amplio abanico de estudio, en varios focos de acción que se vinculan a las relaciones internacionales del Gran Oriente de Italia entre mediados del siglo XIX y comienzos del XX. Para el caso, nos habló de sus averiguaciones entre la mencionada obediencia italiana y sus conexiones con la Gran Logia Unida de Inglaterra (UGLE por sus siglas en inglés) entre 1862 y $1918^{69}$, su aproximación a las comunidades de emigrantes italianos en América, o sus futuras exploraciones en combinación con el Gran Oriente de Francia o la masonería de la Europa del Este (inicialmente en Rumanía). Por otro lado, y además de sus colaboraciones junto al profesor Novarino ${ }^{70}$ antes reseñadas $^{71}$, resalta el interés que tomó por el filósofo, pedagogo y político Carlo Michele Buscalioni y su contribución a la cultura y a la educación desde su membresía masónica ${ }^{72}$.

del siglo XIX. II Symposium de Metodología Aplicada a la Historia de la Masonería Española, coord. Ferrer Benimeli (Valladolid: Consejería de Educación y Cultura, 1987), Tomo I, 247-268.

${ }^{67}$ Uno de los trabajos más relevantes del profesor Kenney en la dirección del tema que se expone es la monografía que se indica a continuación. Véase, James Scott Kenney, Brought to Light: Contemporary Freemasonry, Meaning, and Society (Ontario: Wilfrid Laurier University Press, 2016). Obra la cual fue reseñada en esta revista por el profesor Martínez Moreno: véase, Carlos Francisco Martínez Moreno, "Reseña de Brought to Light", REHMLAC+ 8, no. 2 (diciembre 2016-abril 2017): 333-338, doi: https://doi.org/10.15517/rehmlac.v8i2.27257

68 Véase su perfil científico para recabar más información en: "Demetrio Xoccato", Academia.edu, http://independent.academia.edu/DemetrioXoccato

${ }^{69}$ Véase, Demetrio Xoccato, "Friendship and prejudice: the relations between the UGLE and the Gran Orient of Italy (1862 - 1918)", en Reflections on 300 years of freemasonry, 505-516.

${ }^{70}$ Véase, Novarino y Xoccato, Ariodante Fabretti. Un laico tra impegno politico-sociale e ricerca scientifica (Turín: Fondazione Ariodante Fabretti - Università Popolare di Torino, 2013).

${ }^{71}$ Véase la bibliografía.

72 Véase, Xoccato, "I progetti geopolitici della massoneria filocavouriana: l'azione di Carlo Michele Buscalioni (1864-1885)", en Società Segrete nel Mediterraneo, ed. Locci (Roma: Bastogi, 2014), 75-100. Y también algunas alusiones que se realizan en relación a la acción educativa del Gran Oriente de Italia en una franja histórica determinada: véase, Xoccato, "Il Grande Oriente d'Italia e l'educazione: l'azione delle logge 
En la cercanía de terminar esta primera parte, y tras las consideraciones del profesor Novarino en torno a la gran potencialidad que contuvieron las distintas aportaciones de los jóvenes investigadores, prosiguió el desarrollo de la sesión con la conferencia plenaria titulada Introduction to the Library and Museum of Freemasonry, London, de la doctora Susan Snell, archivera de dicha institución ${ }^{73}$, la cual, además de explicar la información administrativa de su unidad de investigación, proporcionó una serie de datos sobre los recursos al servicio de las posibles pesquisas orientadas hacia la historia de la institución masónica. A modo de ejemplo, habló del Lane's Masonic Records ${ }^{74}$ y cómo extrajo algunos ejemplos de masones italianos que trabajaron o vivieron o tuvieron algún tipo de vínculo completo o parcial con la Inglaterra de los rangos de fechas propuestos. Para el caso, ilustró su referencia con algunos apuntes biográficos de Bartholomew Ruspini, Domenico Santiero y Louis Pilloti, donde se evidenció la importancia de un recurso electrónico que progresivamente irá evolucionando y mejorando a lo largo del presente año.

Clausuró esta parte del evento D. Stefano Bisi, gran maestro del Gran Oriente de Italia, el cual alabó tanto la importancia de la solidaridad histórica de Turín, canalizada en múltiples ocasiones a través de la masonería, como el papel destacado que debe tener la cultura y la investigación histórica en oposición a la ignorancia y las ideas antimasónicas. Un refrendo necesario y alentador para seguir adelante en la tarea que congregó a este elenco de jóvenes investigadores.

Ya en la segunda parte del seminario, tomaron la palabra parte de los miembros asistentes del comité científico, para finalmente concluir sobre las siguientes ideas, muchas de ellas coincidentes:

a) Se incide en el destacado papel de establecer los estudios sobre la masonería como una categoría científica dentro del ámbito universitario.

b) La importancia de digitalizar las fuentes masónicas, como ejemplo de transparencia y de fomento de la cultura.

c) Darle estabilidad a este tipo de encuentros científicos, como foro de intercambio de ideas relevantes.

d) Creación de una revista científica en línea, entroncada con los estudios multidisciplinares que engloban a la propia masonería y que se vea enriquecida con las aportaciones de los nuevos estudios.

nelle grandi città (1868-1925)", REHMLAC+ 9, no. 1 (mayo-noviembre 2017): 56-75, doi: https://doi.org/10.15517/rehmlac.v9i1.28189

${ }_{73}$ Véase en: "The Library and Museum of Freemasonry", The Library and Museum of Freemasonry, http://freemasonry.london.museum/library-museum-archive/

${ }_{74}$ Más información en: véase, "Lane's Masonic Records", The Library and Museum of Freemasonry, http://freemasonry.london.museum/resources-information/lanes-masonic-records/ 
e) Fomentar la visibilidad ante la sociedad civil, junto a una vertiente divulgativa y con especial realce hacia aquellas actividades artísticas y deportivas.

f) Instaurar lazos de investigación entre los distintos países y sus diversas temáticas comunes y propias, creando amplias redes de conocimiento.

g) Preservar el patrimonio histórico de la masonería, siempre abierto a la investigación, incidiendo en la riqueza italiana aún por explorar en profundidad.

h) Incentivar la ayuda a los jóvenes investigadores en vínculo a la historiografía masónica.

i) Potenciar la creación de una red europea interuniversitaria en la que se amplíe el papel de la masonería en conexión con los importantes hitos históricos de Europa.

\section{Conclusión}

En definitiva, un doble deseo: que sigan existiendo foros académicos como este, inclusive próximas ediciones tan bien planteadas y organizadas desde el Centro di Ricerche Storiche sulla Libera-Muratoria (CRSL-M), y más cuando como colofón final se presentó el volumen primero ${ }^{75}$ de los Quaderni del CRSL-M, de la serie Fondi e fonti que su director tuvo a bien presentar en vínculo directo con España; y que estos encuentros estimulen a los investigadores a seguir apostando por esta línea argumental que visualice de forma rigurosa y científica el papel de la masonería a lo largo de los distintos contextos, ayudando a reescribir la narrativa histórica, y apostando por el lógico trasvase a la sociedad. Porque recordemos que tras el foro académico, está el propio de la sociedad al completo; y, por tanto, no debemos caer en la inconsciente idea de otros planteamientos científicos, como bien decía el gran Carl Sagan: "Vivimos en una sociedad extremadamente dependiente de la ciencia y la tecnología, en que casi nadie tiene unas mínimas nociones sobre ciencia y tecnología". Cuidemos ese aspecto, cuidemos de la correcta difusión y posterior divulgación del conocimiento.

\section{Bibliografía}

Anaya Gámez, Fernando M. "Música y masonería: prontuario para un estado de la cuestión" REHMLAC+ 6, no. 1 (mayo-noviembre 2014): 141-145, doi: http://dx.doi.org/10.15517/rehmlac.v6i1.15231

Anaya Gámez, Fernando M. "Y la lira volvió a sonar: breve estudio sobre las relaciones semánticas entre música y masonería". En La masonería hispano-lusa: de los absolutismos a las democracias. XIV Symposium Internacional de Historia de la

\footnotetext{
75 Novarino, Fondi massonici in lingua italiana del Centro Documental de la Memoria Histórica di
} Salamanca (Turín: Centro di Ricerche Storiche sulla Libera-Muratoria, 2017). 
Masonería Española. Coordinado por José Miguel Delgado Idarreta e Yván Pozuelo Andrés. Oviedo: Universidad de Oviedo y CEHME, 2017.

Ayala, José Antonio. "Sociología de la masonería en la región de Murcia". En La masonería en la España del siglo XIX. II Symposium de Metodología Aplicada a la Historia de la Masonería Española. Coordinado por José Antonio Ferrer Benimeli. Valladolid: Consejería de Educación y Cultura, 1987.

Beaurepaire, Pierre-Yves. Nobles jeux de l'Arc et loges maçonniques dans la France des Lumières. Montmorency: Ivore-clair, 2002.

Berger, Joachim. “«Une œuvre internationale d'un caractère humanitaire»: The Appeal to Humanity in International Masonic Relations". En Humanity. A History of European Concepts in Practice From the Sixteenth Century to the Present. Editado por Fabian Klose y Mirjam Thulin. Göttingen: Vandenhoeck \& Ruprecht, 2016.

Casano, Nicoletta. "Les réseaux unissant francs-maçons et laïques belges et italiens de la fin du XIXe siècle jusqu'à la Deuxième guerre mondiale. Prémisses et réalisation de l'accueil en Belgique des fuorusciti italiens". Revue Belge d'Historie Contemporaine XLIII, no. 2/3 (2013): 238-240.

Casano, Nicoletta. Libres et persécutés. Francs-maçons et lä̈ques italiens en exil pedant le fascisme. París: Classiques Garnier, 2016.

Conti, Fulvio. Italia immaginata. Sentimenti, memorie e política fra Otto e Novecento, Pisa: Pacini, 2017.

Conti, Fulvio, Massoneria e religioni civil. Cultura laica e liturgie politiche fra XVIII e XX secolo. Bolonia: Il Mulino, 2008.

Conti, Fulvio. Storia della massoneria italiana. Dal Risorgimento al fascismo. Bolonia: Il Mulino, 2003.

Côrte Real de Camargo, Felipe. "Protect the Integrity: regularidade no discurso das relações maçônicas internacionais entre Brasil e Inglaterra (1880-2000)". REHMLAC+ 8, no. 1 (mayo 2016-noviembre 2016): 130-150, doi: https://doi.org/10.15517/rehmlac.v8i1.24276

Côrte Real de Camargo, Felipe. "Una mirada distinta a las fuentes en la historia de la masonería”, REHMLAC+ 8, no. 2 (diciembre 2016-abril 2017): 307-310, doi: http://dx.doi.org/10.15517/rehmlac.v8i2.27252

Côrte Real de Camargo, Felipe. "La cultura material como fuente para la historia de la masonería: el caso de los mandiles de la masonería inglesa preunificación (1720's1820’s)". En 300 años: Masonerías y masones (1717-2017). Tomo V: Cosmopolitismos. Editado por Ricardo Martínez Esquivel, Yván Pozuelo y Rogelio Aragón. Ciudad de México: Palabra de Clío, 2017.

Delogu, Giulia. La poetica della virtù. Comunicazione política e rappresentazione del potere in Italia tra Sette e Ottocento. Milán - Udine: Mimesis, 2017. 
Delogu, Giulia. "Di virtù lira sonante». Poesia e massoneria in Italia tra Settecento e Primo Ottocento. Pavía: Varini Publishing, 2014.

Fedele, Santi. La Massoneria italiana nell'esilio e nella clandestinità 1927-1939. Milán: Franco Angeli, 2005.

Fedele, Santi. Alessandro Tedeschi Gran Maestro dell'esilio. Bolonia: Percosi, 2008.

Fedele, Santi. La Massoneria italiana tra Otto e Novecento. Foggia: Bastogi Editrice Italiana, 2011.

Ferrer Benimeli, José Antonio. "El modelo sociológico de la masonería". En Masonería, revolución y reacción. IV Symposium Internacional de Historia de la Masonería Española. Coordinado por José Antonio Ferrer Benimeli. Zaragoza: CEHME, 1990.

Fressin, Thomas. Grand Armorial des Chevaliers Bienfaisants de la Cité Sainte. París: autoedición, 2017.

Kenney, James Scott. Brought to Light: Contemporary Freemasonry, Meaning, and Society. Ontario: Wilfrid Laurier University Press, 2016.

Locci, Emanuela. "Las logias italianas en Turquía: entre el fortalecimiento de la comunidad italiana y el cosmopolitismo". REHMLAC+ 9, no. 2 (diciembre 2017 -abril 2018): 47-55, https://revistas.ucr.ac.cr/index.php/rehmlac/article/view/30729/31656

Locci, Emanuela. "The First Lodge in the Ottoman Empire: Oriental Lodge, 1856 -1909". En Reflections on 300 years of freemasonry. Editado por John S. Wade. Londres: Lewis Masonic, 2017.

Locci, Emanuela. Storia della massoneria femminile. Dalle corporazioni alle obbedienze. Roma: BastogiLibri, 2017.

Locci, Emanuela. Massoneria nel Mediterraneo. Egitto, Tunisia e Malta. Roma: BastogiLibri, 2014.

Locci, Emanuela. Il cammino di Hiram. La massoneria nell'Imperio Ottomano. Foggia: Bastogi Editrice Italiana, 2013.

Manenti, Luca G. Massoneria e irredentismo. Geografia dell'associazionismo patriottico in Italia tra Otto e Novecento. Trieste: Irsml FVG, 2015.

Martín Martínez, Luis P. "La Asociación Masónica Internacional (1901-1940) o la utopía pacifista". En La masonería en la España del siglo XX. VII Symposium Internacional de Historia de la Masonería Española. Coordinado por José Antonio Ferrer Benimeli. Zaragoza: CEHME, Universidad de Castilla-La Mancha, Cortes de Castilla-La Mancha, 1996.

Martínez Esquivel, Ricardo e Yván Pozuelo Andrés. "Educación escolar y masonería: krausismo y laicidad entre España y Costa Rica a finales del siglo XIX". REHMLAC+ 9, No. 1 (mayo-noviembre 2017): 127-145, doi: http://dx.doi.org/10.15517/rehmlac.v9i1.28631 
Martínez Moreno, Carlos Francisco. "Reseña del libro Brought to Light de James Scott Kenney”. REHMLAC+ 8, no. 2 (diciembre 2016-abril 2017): 333-338, doi: https://doi.org/10.15517/rehmlac.v8i2.27257

Miletto, Enrico. “...la coltura per il popolo”. L’Università Popolare di Torino (19001930). Turín: Università Popolare di Torino Editore, 2013.

Miletto, Enrico y Marco Novarino. "Senza distinzione política e religiosa”. Repertorio bibliográfico e archivístico sull'associazionismo laico in Piemonte (1848-1925). Turín: Centro Studi Piero Calamandrei, 2013.

Miletto, Enrico y Marco Novarino. "Senza distinzione politica e religiosa”. Repertorio bibliográfico e archivístico sull'associazionismo laico a Torino e provincia (18481925). Turín: Centro Studi Piero Calamandrei, 2011.

Mola, Aldo Alessandro. "Las relaciones masónicas italiano-españolas y la Avocación Masónica Internacional”. En La masonería en la España del siglo XX. VII Symposium Internacional de Historia de la Masonería Española. Coordinado por José Antonio Ferrer Benimeli. Zaragoza: CEHME, Universidad de Castilla-La Mancha, Cortes de Castilla-La Mancha, 1996.

Morelli, Anne. La participation des émigrées italiens à la résistance belge. Roma: Ministerio Affari Esteri, 1983.

Morelli, Anne. Fascismo e antifascismo nell'emigrazione italiana in Belgio: 1922-1940. Turín: Bonacci, 1987.

Novarino, Marco y Demetrio Xoccato. Ariodante Fabretti. Un laico tra impegno politicosociale e ricerca scientifica. Turín: Fondazione Ariodante Fabretti - Università Popolare di Torino, 2013.

Novarino, Marco. Fondi massonici in lingua italiana del Centro Documental de la Memoria Histórica di Salamanca. Turín: Centro di Ricerche Storiche sulla LiberaMuratoria, 2017.

Pozuelo Andrés, Yván. "Kipling y su sorprendente primera novela”, REHMLAC+ 5, no. 2 (diciembre 2013-abril 2014):

$143-160$, https://revistas.ucr.ac.cr/index.php/rehmlac/article/view/12939/12235

Pozuelo Andrés, Yván. Guía histórica de la masonería en Asturias. Oviedo: Masónica, 2015.

Xoccato, Demetrio. "Friendship and prejudice: the relations between the UGLE and the Gran Orient of Italy (1862-1918)". En Reflections on 300 years of freemasonry. Editado por John S. Wade. Londres: Lewis Masonic, 2017.

Xoccato, Demetrio. "I progetti geopolitici della massoneria filocavouriana: l'azione di Carlo Michele Buscalioni (1864-1885)”. En Società Segrete nel Mediterraneo. Editado por Emanuela Locci. Roma: Bastogi, 2014. 
Xoccato, Demetrio. "Il Grande Oriente d'Italia e l'educazione: l'azione delle logge nelle grandi città (1868-1925". REHMLAC+9, no. 1 (mayo-noviembre 2017): 56-75, doi: https://doi.org/10.15517/rehmlac.v9i1.28189 